\title{
Associação entre aspectos depressivos e déficit visual causado por catarata em pacientes idosos
}

\author{
Association of depressive aspects with visual impairment caused by cataract in the elderly
}

\author{
João Eduardo Caixeta Ribeiro' \\ Michelle Márcia de Freitas ${ }^{2}$ \\ Gilberto de Sousa Araújo ${ }^{3}$ \\ Tiago Humberto Rodrigues Rocha ${ }^{4}$
}

Trabalho realizado no Instituto de Ciências Biológicas e da Saúde.

Subvencionado por verba destinada ao Programa de Iniciação Científica da UNIUBE - PBIC UNIUBE ICBS $012 / 00$.

${ }^{1}$ Doutor em Medicina; Professor do Instituto de Ciências Biológicas e da Saúde da Universidade de Uberaba - UNIUBE.

${ }^{2}$ Psicóloga; ex-aluna do Programa de Iniciação Científica da Universidade de Uberaba.

${ }^{3}$ Mestre em Estatística; Professor do Instituto de Ciências Biológicas e da Saúde da Universidade de Uberaba - UNIUBE.

${ }^{4}$ Acadêmico de Psicologia; Aluno de Iniciação Científica da Universidade de Uberaba.

Endereço para correspondência: João Eduardo Caixeta Ribeiro, Av. Santos Dumont, 409 - Uberaba (MG) CEP 38060-360

E-mail: joaoeduardo@enetec.com.br

Recebido para publicação em 16.12.2003

Versão revisada recebida em 06.05.2004

Aprovação em 17.05.2004

\section{RESUMO}

Objetivo: Investigar a associação entre a presença de sintomas depressivos e a deficiência visual causada por catarata no paciente idoso. Métodos: Vinte e três pacientes com catarata e acuidade visual inferior a 20/200 no melhor olho foram avaliados. As idades variavam de 60 a 93 anos. Antes da cirurgia de catarata e um mês após, os sintomas depressivos foram avaliados pela Escala de Depressão Geriátrica (EDG). Resultados: A cirurgia melhorou a visão para 20/50, ou melhor, em todos os pacientes. Antes e após a cirurgia de catarata foram encontrados $11(47,82 \%)$ e $10(43,47 \%)$ indivíduos com escores indicativos de depressão respectivamente ( $\mathrm{p}=1,0$; teste de McNemar). Antes da cirurgia observamos o valor mediano daEDG de 5,0 e após a cirurgia a EDG apresentou o valor mediano de 4,0 ( $\mathrm{p}=0,012$; Wilcoxon pareado). Neste período os sintomas indicativos de depressão diminuíram significativamente, de valores entre 3 a 8 pontos para valores entre 3 a 6 pontos. Conclusões: Sintomas depressivos são prevalentes e persistentes entre pacientes idosos, entretanto os níveis dos sintomas indicativos de depressão diminuem significativamente com a melhora da visão.

Descritores: Catarata; Facoemulsificação; Depressão; Transtorno depressivo/diagnóstico; Transtorno depressivo/etiologia; Avaliação geriátrica; Escala de graduação psiquiátrica; Transtornos visuais; Atividades cotidianas; Idoso

\section{INTRODUÇÃO}

A Organização Mundial da Saúde estima que em 2025 o Brasil será o sexto país mais velho do planeta com cerca de 32 milhões de pessoas com mais de 60 anos. A deterioração de alguns sistemas orgânicos caracterizam a idade provecta. O declínio biológico é inevitável, já que todos os sistemas do corpo decaem tanto nos aspectos estruturais como funcionais. Muitos idosos perdem muito do seu poder cognitivo e perceptivo ${ }^{(1-4)}$.

A perda visual desencadeia um processo de alteração emocional e psicológica que se caracteriza basicamente por desordem de ajustamento e depressão ${ }^{(5)}$. Estas alterações geralmente são acompanhadas por incapacidades para as atividades do dia a $\operatorname{dia}^{(1)}$. No idoso, a diminuição da capacidade visual geralmente se deve a doenças oculares crônicas que vão diminuindo a visão de maneira progressiva ${ }^{(6-7)}$. Problemas oftalmológicos estão associados a altas taxas de depressão e a dificuldades para as atividades diárias, levando a condutas inadequadas uma vez que sintomas de depressão se assemelham a sintomas relacionados às alterações nas atividades diárias e vice-versa ${ }^{(8)}$.

A relação entre a depressão e o problema clínico é complexa. A doença clínica pode precipitar um distúrbio depressivo preexistente. Certas doenças podem ser acompanhadas das chamadas depressões "secundárias", ou 
o problema médico pode ser conseqüência de um estado depressivo. Em outros casos, a doença clínica pode mimetizar um quadro depressivo ${ }^{(9)}$.

Em um estudo realizado, foi encontrado $27 \%$ de indivíduos idosos com sintomatologia depressiva, sendo $19 \%$ com quadro de disforia, $4 \%$ com quadro de depressão sintomática, $2 \%$ de distimia, $1,2 \%$ de síndrome depressiva ansiosa e $0,8 \%$ de depressão maior ${ }^{(3)}$. O reconhecimento da depressão na idade avançada pode ser mais difícil do que em idades anteriores. Nessa faixa etária, tanto o clínico quanto o próprio paciente podem atribuir a depressão ao processo de envelhecimento.

Em geral, a mudança de "status" social do idoso, que implica na diminuição de recursos, no crescimento da dependência familiar e, a obrigação de deixar o lugar habitual de vida tornam-se fatores contribuintes para aparição de uma depressão. E, dentre os diferentes modelos, a teoria médica cita o aparecimento de doenças crônicas, a redução da sensorialidade e as incapacidades múltiplas como fatores depressores ${ }^{(2)}$.

O presente trabalho teve como objetivo investigar a associação entre a presença de aspectos depressivos e a deficiência visual no idoso com catarata, tratando estes casos cirurgicamente e avaliando o impacto da melhora da visão nas alterações depressivas. Avaliou ainda, a correlação entre déficit visual e alterações funcionais para atividades básicas diárias.

\section{MÉTODOS}

Todas as fases deste trabalho seguiram as exigências éticas e científicas contidas na resolução 196/96 do Conselho Nacional de Saúde (CNS) e seu início se deu após avaliação e aprovação pelo Comitê de Ética em Pesquisa da Universidade de Uberaba.

Vinte e três (23) indivíduos com idade acima de 60 anos e com catarata, participantes do Programa Nacional de Prevenção à Cegueira, foram selecionados para o presente trabalho, através de triagem utilizando-se o exame da acuidade visual. Foi avaliado cada olho em separado, utilizando-se a tabela de Snellen a seis metros (E chart), que permite a aferição da visão em indivíduos alfabetizados ou não. Aqueles pacientes que apresentaram visão inferior a 20/200 no melhor olho foram submetidos à consulta oftalmológica completa incluindo nova medida da acuidade visual, refração, exame do fundo de olho, tonometria e biomicroscopia. Os pacientes não incluídos no presente trabalho receberam correção ótica ou foram encaminhados para acompanhamento oftalmológico seguindo a rotina da Secretaria Municipal de Saúde de Uberaba.

Após a exposição dos riscos e benefícios envolvidos na presente pesquisa, esclarecimentos relativos à pesquisa tais como justificativa, objetivos, procedimentos a serem realizados, existência ou não de métodos alternativos e preenchimento de consentimento livre e esclarecido segundo a resolução 196/96 do CNS, deu-se prosseguimento ao trabalho e os pacientes foram avaliados do ponto de vista psicológico através da Escala de Depressão Geriátrica - EDG(Geriatric Depression Scale) ${ }^{(10)}$. A escala de depressão geriátrica é um dos mais utilizados instrumentos para detecção de depressão em idosos e estudo recente demonstrou que a versão brasileira do EDG-15 (escala com 15 perguntas) oferece medida válida para o diagnóstico de episódio depressivo e o ponto de corte 5/6 (não caso/caso) produz índice de sensibilidade de $85,4 \%$ e especificidade de $73,9 \%$ para o diagnóstico destes episódios ${ }^{(11)}$. Considerou-se que o paciente não apresentava depressão com escores menores ou iguais a $5(E D G \leq 5)$ e indicativos de depressão com escores acima de 6.

Antes da cirurgia de facoemulsificação os pacientes foram submetidos à avaliação clínica completa, exames laboratoriais e após liberação pelo médico clínico-geral (cardiologista) foram submetidos à cirurgia proposta.

As cirurgias monoculares foram realizadas por médico oftalmologista, sob anestesia local e utilizando-se o método da facoemulsificação com implante de lente intra-ocular de câmara posterior. Após a recuperação visual, em média 30 dias após a cirurgia, os pacientes foram submetidos à nova avaliação psicológica utilizando-se a mesma escala (EDG), para que os resultados pudessem ser reavaliados e comparados.

Os pacientes foram avaliados pelo EDG e pelo Índice de Independência em Atividades de Vida Diária que avalia as atividades funcionais diárias básicas e a mobilidade dos indivíduos. Dados obtidos pelo índice de independência em atividades de vida diária receberam o tratamento proposto por $\mathrm{Katz}^{(12)}$, avaliando o grau de Dependência ou Independência do Idoso. Respostas A: Independente em todas as funções; B: Uma das funções; C: Dependente em tomar banho mais uma função; D: Dependente em tomar banho, vestir-se, higiene corporal e mais outra função; F: Dependente em tomar banho, vestir-se, higiene corporal, locomoção e outra função, controle da continência urinária fecal ou alimentação; G: Dependente em todas as funções; H: Outros - dependente em pelo menos duas funções, mas não classificadas em C, D, E ou F.

\section{ANÁLISE ESTATÍSTICA}

Para verificar se a cirurgia de catarata influenciou na intensidade da escala de depressão geriátrica, foi utilizado o teste não paramétrico de Wilcoxon pareado, enquanto que para analisar se o paciente apresentava depressão $(E D G>5)$ ou não apresentava depressão (EDG $\leq 5)$ e independência em atividades de vida diária antes e após a cirurgia de catarata utilizamos o teste de Mc Nemar. O indicativo de depressão entre os sexos tanto no pré quanto no pós-operatório foi estudado a partir do Teste Exato de Fisher. A suposição de normalidade dos dados foi realizada a partir do teste de Kolmokorov-Smirnov, sendo os dados não normais apresentados por mediana (mínimo; máximo). O nível de significância para todos os testes foi considerado $\mathrm{p}<0,05$. 


\section{RESULTADOS}

Vinte e três pacientes com acuidade visual inferior a 20/200 causada por catarata foram avaliados pelo EDG e submetidos à cirurgia. Destes, $12(52,17 \%)$ eram do sexo masculino e 11 $(47,82 \%)$ do sexo feminino, com idades variando entre 60 e 93

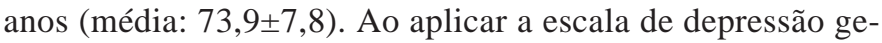
riátrica, antes da cirurgia de catarata, foram encontrados 11 $(47,82 \%)$ indivíduos com escores indicativos de depressão. Destes $3(27,27 \%)$ eram do sexo masculino e $8(72,72 \%)$ do feminino. Após a cirurgia foram encontrados $10(43,47 \%)$ indivíduos com escores indicativos de depressão, sendo 2 (20\%) do sexo masculino e $8(80 \%)$ do sexo feminino.

Analisando a intensidade da escala de depressão geriátrica observamos que após a cirurgia de catarata houve uma redução significativa na intensidade desta escala $(p=0,012)$. Antes da cirurgia observamos um valor mediano da EDG de 5,0 (min 2,0; $\max 11,0)$ e após a cirurgia a EDG apresentou um valor mediano de 4,0 $(\min 2,0 ; \max 11,0)$ (Figura 1).

Tanto no pré-operatório (Figura 2 A) quanto no pós-operatório (Figura 2 B) a proporção de mulheres deprimidas foi significativamente superior a proporção de homens deprimidos $(\mathrm{p}=0,03 \mathrm{e}$ $\mathrm{p}=0,01$ ).

Não observamos no grupo masculino e no grupo feminino uma diferença significativa da proporção de indicativo de depressão entre o pré e o pós-operatório (teste $\mathrm{McNemar}, \mathrm{p}=1,0$ $\mathrm{e} \mathrm{p}=0,48)$, respectivamente.

Analisando a intensidade da escala de depressão geriátrica no sexo masculino e no feminino antes e após a cirurgia de catarata, observamos diferença significativa na intensidade

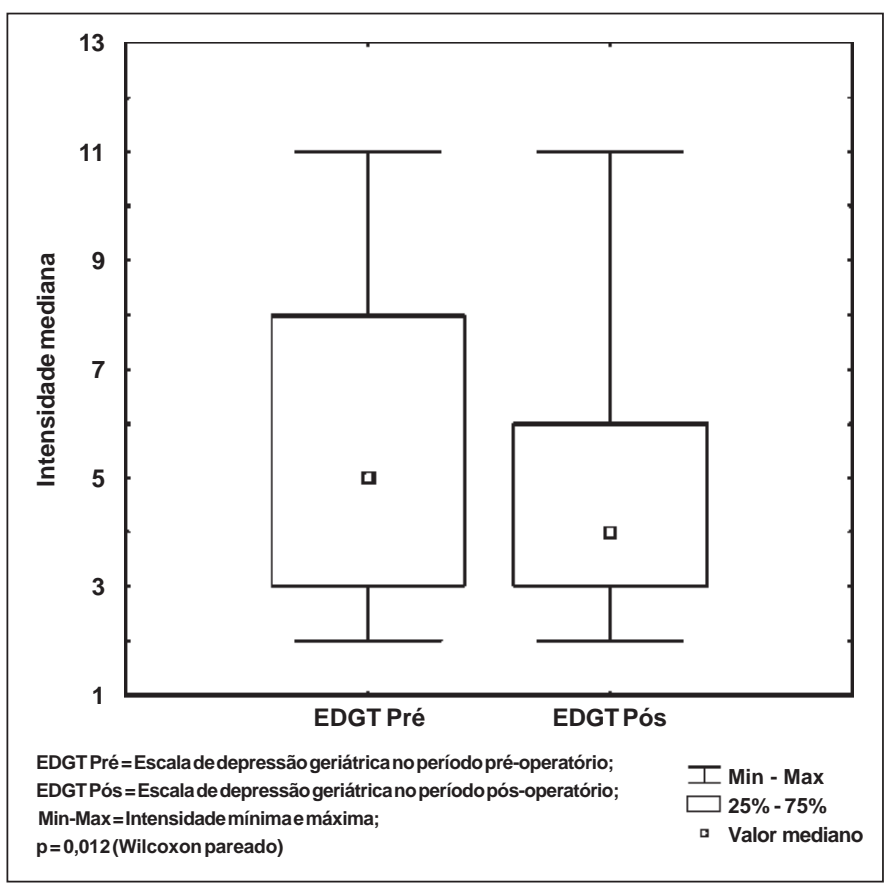

Figura 1 - Intensidade mediana da escala de depressão geriátrica antes e após a cirurgia de catarata na amostra estudada

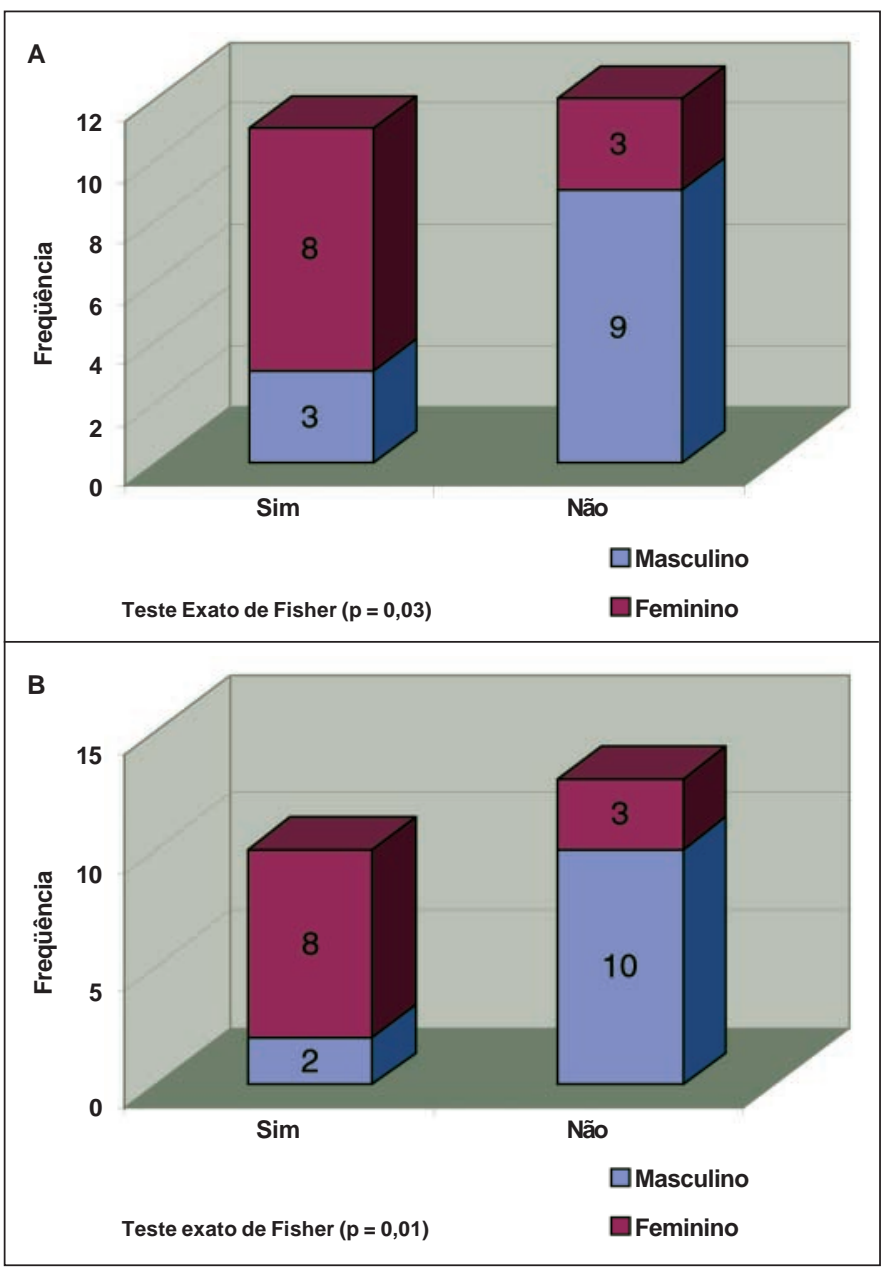

Figura 2 - Distribuição de casos de depressão geriátrica segundo o sexo; A - no pré-operatório e B - no pós-operatório

somente no grupo feminino, com redução do indicativo de depressão no pós-operatório ( $\mathrm{p}=0,049)$ (Figura 3 ).

Analisando o índice de independência em atividades de vida diária verificamos que todos os indivíduos eram independentes nas funções avaliadas tanto no período pré-operatório quanto no pós-operatório.

\section{DISCUSSÂO}

Para países em desenvolvimento a OMS define terceira idade como sendo aquele grupo de indivíduos com mais de 60 anos e é nesta faixa etária que a prevalência de doenças oculares aumenta ${ }^{(6)}$.

Neste estudo encontramos correlação entre dificuldade visual e níveis indicativos de depressão nos pacientes com história clínica de catarata estando estes resultados em concordância com outros estudos já realizados ${ }^{(14-15)}$. As atividades funcionais diárias básicas e a mobilidade dos indivíduos, obtidos pelo índice de independência em atividades de vida diária, receberam o tratamento proposto por Katz ${ }^{(12)}$. Tanto no 


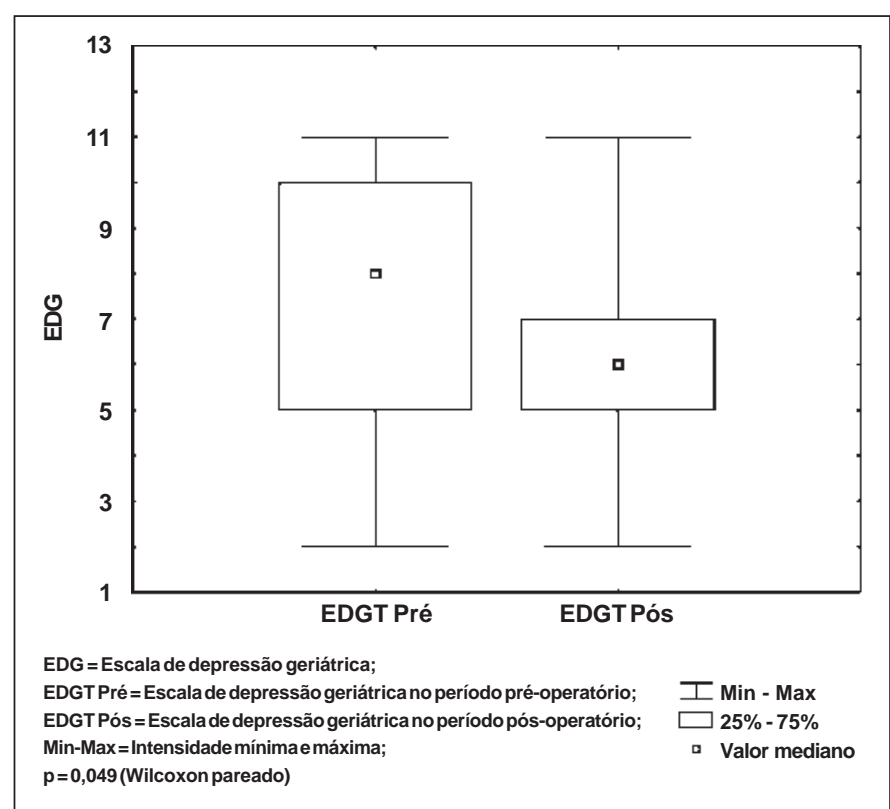

Figura 3 - Intensidade mediana da escala de depressão geriátrica antes e após a cirurgia de catarata no grupo feminino

período pré-operatório quanto no pós-operatório todos os pacientes foram classificados como independente em todas as funções. Sabe-se que a diminuição progressiva e gradual da visão leva a uma adaptação dos pacientes e as atividades avaliadas por este índice (tomar banho, vestir-se, higiene corporal, controle da continência urinária fecal ou alimentação) podem ser adaptadas à condição de pouca visão principalmente no indivíduo aposentado e com poucas atividades diárias.

A dificuldade visual dos pacientes, sendo todos diagnosticados com catarata, foi avaliada apenas pela acuidade visual à distância e outras alterações visuais tais como baixa acuidade visual para perto, alteração na visão de cores, no campo visual, na sensibilidade ao contraste e na percepção de profundidade poderiam estar relacionados com a situação destes pacientes. $\mathrm{O}$ aspecto emocional foi abordado somente de maneira quantitativa e qualitativa sem uma confirmação clínica da depressão, e outros problemas físicos ou sociais que pudessem estar relacionados às alterações do humor não foram correlacionados $^{(1)}$. Por outro lado a intervenção cirúrgica e os procedimentos médicos seguintes, mesmo que simples tecnicamente, podem ser considerados como fatores estressantes e fonte de depressão(13).

Estudando problemas visuais em diabéticos constatou-se que as alterações psicossociais são compensadas quando o déficit visual é estável ${ }^{(7)}$. Em outro estudo foi observado que a maioria dos pacientes, avaliados sobre o impacto psicológico da catarata, evidenciaram um processo de adaptação à situação de deficiência visual ${ }^{(14)}$, fato também observado no presente estudo principalmente nas atividades de vida diária.

A escala de depressão geriátrica (EDG) aplicada neste estudo é mundialmente aceita ${ }^{(8,15-17)}$ para o diagnóstico de epi- sódio depressivo. Entretanto a maioria das escalas aplicadas com este objetivo falha no acesso à história psiquiátrica dos idosos demonstrando a necessidade de uma escala validada para este grupo etário específico ${ }^{(17)}$

O principal objetivo deste estudo foi investigar a associação entre aspectos depressivos e déficit visual causado por catarata em pacientes idosos. Encontramos diferença significativa na intensidade da escala de depressão geriátrica neste grupo após a melhora da visão. Isto está em concordância com a literatura que demonstra que a melhora visual leva a melhora do quadro depressivo(13-14). Nestes quadros a dimensão médico-social tem importância crucial considerando as perdas cognitivas, físicas, econômicas e sociais. Há uma complexidade de problemas nos níveis psíquico, somático e social que determinam a progressiva perda da capacidade de adaptação do indivíduo ao meio ambiente. $\mathrm{O}$ auxílio de uma psicoterapia favorece a integração ao meio e a motivação para adaptar-se a ele.

\section{CONCLUSÃO}

A dificuldade visual encontrada nos idosos é fruto de um processo fisiológico com progressiva redução da reserva funcional que, embora geralmente camuflados nesta faixa etária, merecem atenção diagnóstica e terapêutica proporcionando melhoria na qualidade de vida desses indivíduos.

Portanto, quando comparamos a intensidade da escala de depressão geriátrica antes e após a cirurgia verificamos uma diminuição significativa desta escala, evidenciando uma associação entre aspectos depressivos em pacientes acima de 60 anos com baixa acuidade visual causada por catarata e a melhora destas alterações emocionais com o implemento da visão.

\section{ABSTRACT}

Purpose: To investigate the association of depressive symptoms with visual impairment caused by cataract in the elderly. Methods: Twenty-three patients with cataract and visual acuity less than 20/200 were studied. Ages ranged from 60 to 93 years. Before the cataract operation and one month there after the patient's depression was tested using the Geriatric Depression Scale-GDS. Results: The cataract surgery restored visual acuity to $20 / 50$ or better in all patients. Before and after the surgery, $11(47.82 \%)$ and $10(43.47 \%)$ patients had scores indicative of depression, respectively ( $\mathrm{p}=1.0$; McNemar test). The average GDS score for all subjects before operation was 5.0 and after the cataract surgery it was $4.0(\mathrm{p}=0.012$; paired Wilcoxon). After the operation the subjects' depression symptoms had significantly diminished, from 3 to 8 points before to 3 to 6 points after surgery. Conclusion: Depressive symptoms are prevalent and persistent among elderly patients however depression rates decrease with improved visual acuity. 
Keywords: Cataract; Phacoemulsification; Depression; Depressive disorder/diagnosis; Depressive disorder/etiology; Geriatric assessment; Psychiatric status rating scales; Vision disorders; Activities of daily living; Aged

\section{REFERÊNCIAS}

1. Rovner BW, Zisselman PM, Shumely-Dulitzki Y. Depression and disability in older people with impaired vision: a follow-up study. J Am Geriatr Soc. 1996;44(2):181-4.

2. Monfort J. La dépression du sujet âgé: revue bibliographique. Ann Med Psychol.(Paris) 1994;152(8):511-28.

3. Blazer D, Hughes DC, George LK. The epidemiology of depression in an elderly community population. Gerontologist. 1987;27(3):281-7.

4. Rocha FL. Depressão no idoso. J Bras Psiquiatr. 1993;42(4):203-8.

5. Leinhaas MM, Hedstrom NJ. Low vision: How to assess and treat its emotional impact. Geriatrics. 1994;49(5):53-6.

6. José NK, Zacharias LC, Graziano RM, Oliveira BFT, Hatanaka, M, Cresta FB. Manual da boa visão. Faculdade de Ciências Médicas da UNICAMP. Campinas: Gráfica da Universidade Estadual de Campinas; 1983.

7. Bernbaum M, Albert SG, Duckro PN. Psychosocial profiles in patients with visual impairment due to diabetic retinopathy. Diabetes Care. 1988;11(7):551-7.
8. Rovner BW, Ganguli M. Depression and disability associated with impaired vision: The Movies project. J Am Geriatr Soc. 1998;46(5):617-9.

9. Gregory RJ, Jimerson DC, Walton BE, Daley J, Paulsen RH. Pharmacotherapy of depression in the medically ill: directions for future research. Gen Hosp Psychiatry. 1992;14(1):36-42.

10. Yesavage JA, Brink TL, Rose TL, Lum O, Huang V, Adey M, et al. Development and validation of a geriatric depression screening scale: a preliminary report. J Psychiatr Res. 1983;17(1):37-49.

11. Almeida OP, Almeida SA. Confiabilidade da versão brasileira da escala de depressão geriátrica (GDS) versão reduzida. Arq Neuropsiquiatr. 1999;57 (2B):421-6.

12. Katz S, Ford AB, Moskowitz RW, Jackson BA, Jaffe MW. Studies of illness in the aged. The Index of ADL: a standardized measure of biological and psychosocial function. JAMA. 1963;185(12):914-9.

13. Roulier D, Tabatabay C, Roth A, Haynal A. Impact psychologique de l'operation de la cataracte chez le patient âgé. J Fr Ophtalmol. 1991;14(1):5-11.

14. Fagerstrom R. Correlation between psychic and somatic symptoms and vision in aged patients before and after a cataract operation. Psychol Rep. 1991;69(3 Pt 1):707-21.

15. Heller J, Weggemans RM, Ferry M, Guigoz Y. Mental health: minimental state examination and geriatric depression score of elderly Europeans in the SENECA study of 1993. Eur J Clin Nutr. 1996;50(2):112-6.

16. Timiras PS. Development physiology and aging. In: Kenney RA. Physiology of aging, New York: Mcmillan; 1972. part II, p.408-614.

17. Weiss IK, Nagel CL, Aronson MK. Aplicability of depression scales to old person. J Am Geriatr Soc. 1986;34(3):215-8.

\section{ERRATA}

\section{Nos Arq Bras Oftalmol. 2004;67(4):631-5 onde se lê Marcia Maria Motono Chojniak leia-se Marcia Motono.}

